\title{
Planetary Nebulae as Probes of the Chemical Evolution of Dynamically Hot Systems
}

\author{
Michael G. Richer ${ }^{1}$ and Marshall L. McCall ${ }^{2}$ \\ 1 Observatorio Astronómico Nacional, Instituto de Astronomía, UNAM, P.O. Box \\ 439027, San Diego, CA 92143 \\ 2 Department of Physics and Astronomy, York University, 4700 Keele St., Toronto, \\ Ontario, Canada M3J 1P3
}

\begin{abstract}
The measurement of chemical abundances in planetary nebulae in nearby galaxies is now relatively straightforward. The challenge is to use these chemical abundances to infer the chemical evolution of their host galaxies. At this point, our understanding of chemical evolution based upon planetary nebulae in galaxies without star formation is strongly coupled to our understanding of the relationship between the chemical abundances in stars and planetary nebulae in the Milky Way bulge. Supposing that the same relation holds in all systems where star formation ceased long ago, these systems follow a metallicity-luminosity relation that is displaced to higher chemical abundances compared to that found for dwarf irregular galaxies. A more efficient enrichment process appears to be required as part of the explanation for this shift, in addition to the inevitable fading of these galaxies.
\end{abstract}

\section{Introduction}

As a result of its evolution, a galaxy consumes gas to form stars. Given a stellar initial mass function, the stars born in any given generation will have a variety of lifetimes. The most massive stars live only several $10^{6}$ years before exploding as supernovae (SNe), their lifetimes being comparable to the time scale for star formation. When they explode as supernovae, these stars return some fraction of their initial mass to the interstellar medium (ISM). Of this returned mass, some of it will consist of elements synthesized during the star's evolution and so the composition of the ISM will change over time as these contributions accumulate. One consequence is that the stars forming at later times contain higher abundances of these newly-synthesized elements. Most stars contribute to this process of enriching the ISM, though the time scales for the majority of stars to contribute is of order several $10^{9}$ years. The study of the chemical evolution of galaxies attempts to characterize this process of chemical enrichment as a function of galaxy type, mass, and environment to better constrain theories of galaxy formation and evolution.

Dynamically hot systems (DHSs) are stellar systems whose kinematics are dominated by random motions, such as elliptical and dwarf spheroidal galaxies and the bulges of spirals [1]. Generally, DHSs have little gas and their principal phase of star formation ceased several $10^{9}$ years ago. Traditionally, the integrated spectrum of starlight has been used to study the chemical evolution of these 
systems, though the interpretation of integrated spectra is difficult due to the interplay of the metallicities and ages of the stellar population mix in any real galaxy. Nonetheless, it has long been known that these systems define a trend between the strength of their absorption lines, e.g., the $\mathrm{Mg}_{2}$ index, and their stellar velocity dispersions, e.g., $[7,2]$. This trend is believed to arise due to winds that develop as a result of the energy that supernovae inject into a galaxy's ISM while star formation is still underway [21].

The lack of ongoing star formation in DHSs requires that another probe besides H II regions, the usual tool, be used to study their chemical evolution. This is unfortunate, since, in $\mathrm{H}$ II regions, it is possible to measure the present abundances of certain elements whose production appears easy to understand. It is possible to study the chemical evolution of DHSs using stars or planetary nebulae (PNe) as probes, e.g., [41], provided that it is possible to observe elements whose abundances are unchanged as a result of the probe's evolution. These probes have the advantage over integrated spectra that their elemental abundances may be obtained directly from their spectra. The principal disadvantages of stars and $\mathrm{PNe}$, as compared to $\mathrm{H}$ II regions, are determining their ages, and therefore the epoch in a galaxy's evolution to which their chemical composition corresponds, whether their chemical abundances have been altered by stellar evolution, and at what enrichment level the process of chemical evolution ceased in a given galaxy. A wider range of elemental abundances may be determined from the spectra of stars than from those of PNe, but stellar spectra are more difficult to measure to the required precision unless the stars are much brighter than the galaxy background or unless the latter is faint. In the central parts of galaxies, it is easier to obtain spectra of $\mathrm{PNe}$, which stimulates interest in their use for studying the chemical evolution of DHSs.

Recently, [26] reviewed this topic. That review focussed more upon some of the theoretical underpinnings of studying the chemical evolution of DHSs. Here we shall focus more on issues related to observations.

\section{Spectroscopy of extragalactic planetary nebulae}

To date, published data have come from 4m-class telescopes. $8 \mathrm{~m}$-class telescopes should allow the derivation of oxygen abundances from measured electron temperatures for galaxies out to at least $5 \mathrm{Mpc}$, and further if high spectral and spatial resolution are used for measuring [O III $] \lambda 4363$.

A variety of observational difficulties must be confronted when obtaining spectroscopy of extragalactic PNe. The first arises from differential atmospheric refraction over the wide wavelength interval that must be observed $[17,47]$. Multiobject spectrographs are an attractive option to increase the efficiency of the observations by observing many $\mathrm{PNe}$ in a galaxy at once, but many of these instruments lack atmospheric dispersion compensators and, unlike in the case of long slit spectroscopy, it is not possible to orient multi-object spectrographs so that the slitlets align with the parallactic angle. The second difficulty is subtracting the light from the background galaxy, which is often much brighter than 
the sky. The stellar absorption lines coincide with the nebular Balmer lines and can vary in intensity on small spatial scales. If there is an underlying galaxy disk, the diffuse interstellar medium and its small-scale variation further complicate the background subtraction by emitting a nebular spectrum whose line intensities can be dramatically different from those in the PNe under study. Emission lines from such gas preferencially comes from low ionization states, e.g., H I, $\left[\begin{array}{ll}\mathrm{O} I\end{array}\right],[\mathrm{N} \mathrm{II}]$, and $[\mathrm{S} \mathrm{II}]$, but $[\mathrm{O} \mathrm{III}]$ is also detected, though He I frequently is not [12]. Emission from the night sky near He I $\lambda 5876$ and $\mathrm{H} \alpha$ - $[\mathrm{N}$ II] is detrimental too, though it is spatially uniform, in constrast to the galaxy background. [36] provide an instructive discussion of this issue and argue convincingly of the advantages of 3D spectroscopy, especially the advantage of having a $2 \mathrm{D}$ spatial map of the background emission to better subtract this emission. A third difficulty is the weakness of the $[\mathrm{O} \mathrm{III}] \lambda 4363$ line, which is about 100 times fainter than [O III] $\lambda 5007$ [35], and its proximity to the $\mathrm{Hg} \mathrm{I} \lambda 4358$ line at observatories whose night sky emission is contaminated by street lights. [O III] $] 4363$ must be observed if the electron temperature is to be measured and used to obtain a reliable estimate of the oxygen abundance.

It is difficult to characterize the absolute precision of the line intensities measured to date in extragalactic PNe and the chemical abundances derived from them. The most extensive comparison may be made using the PNe studied in M32 by [35,32], who used the same equipment and refinements of the same observational technique. For lines stronger than $\mathrm{H} \beta$, the uncertainty appears to be about $\pm 25 \%$. For lines whose strength is $50 \%$ that of $\mathrm{H} \beta$, the uncertainty appears to be about a factor of two. However, in all cases, the abundances derived in both studies agree within uncertainties. These "internal" uncertainties can be as low as $\pm 15 \%$ and \pm 0.1 dex for $\mathrm{He}$ and $\mathrm{O}$ abundances, respectively, but may underestimate the true uncertainties if they do not include all systematic uncertainties.

There are few observations of a given object by different groups. In M31, PN29 is common to three studies. The values found for the oxygen abundance, the $\mathrm{Ne} / \mathrm{O}$ ratio, and the $\mathrm{N} / \mathrm{O}$ ratio all span a range of about 0.3 dex $[35,17,36]$. The helium abundance differs by almost $40 \%$ between two of the studies $[17,36]$. PN23 has been observed twice and the oxygen abundances and Ne/O ratios agree within uncertainties [35,17]. PN27 has also been observed twice [17,36], with the resulting oxygen abundances in excellent agreement, but the helium abundances differing by $35 \%$. Although it is an indirect indicator, there are several detections of He I $\lambda 5876$ in PNe in M31 that are either anomalously strong or weak, perhaps as a result of significant background contamination [35,17]. In M32, the line intensities for PN1 are in generally good agreement among the available measurements $[18,35,32]$. Considering all of the data available, the case of PN29 in M31 appears unusual. Were the oxygen abundances generally as uncertain as those measurements imply, dispersions in abundances within a given galaxy should be at least $0.3 \mathrm{dex}$. In M32, NGC 185, and NGC 205, the dispersions in the oxygen abundances for PNe with measured electron temperatures are 0.19 dex, $0.27 \mathrm{dex}$, and $0.37 \mathrm{dex}$, respectively [35,32]. A dispersion as low as that found 
for M32 (13 PNe) implies that the typical uncertainty in oxygen abundances should be less than 0.2 dex, which is comparable to the uncertainties quoted for individual abundances (when quoted).

It appears that background subtraction may be the dominant source of uncertainty in the line intensities at the moment [36]. Currently, there are too few measurements to determine whether the differences among studies are systematic. Helium abundances appear to be uncertain by up to about $40 \%$, while oxygen abundances may be uncertain by up to $60 \%$. Abundance ratios of neon and nitrogen relative to oxygen may be less uncertain, but one can anticipate difficulties with the background subtraction for nitrogen lines (and eventually those of sulphur). In principle, observations with higher spectral resolution should help, since the PNe should have velocities that scatter about the velocity of the diffuse ionized gas in the ISM, allowing separation of their components.

What matters, however, for studies of the chemical evolution of galaxies are the mean abundances and their dispersions for a population of PNe. Provided that the oxygen abundances for individual PNe in current studies do not deviate systematically from their true values, the mean value for a sample of $\mathrm{PNe}$ should be robust. On the other hand, the dispersions in abundances may be more seriously affected if the true uncertainties are severely underestimated.

\section{Interpreting the chemical abundances in bright planetary nebulae}

The first issue that has an important bearing upon the interpretation of the abundances measured in $\mathrm{PNe}$ is the object selection process. To derive the most secure oxygen abundances, the electron temperature must be measured, requiring that $[\mathrm{O}$ III $] \lambda 4363$ be detected. Since this line is about 100 times fainter than $[\mathrm{O}$ III $] \lambda 5007$, the sample will normally be restricted to PNe that are bright in $[\mathrm{O}$ III $]$ 25007. "Bright PNe" is adopted to denote PNe bright in $[\mathrm{O}$ III $] \lambda 5007$, usually within $2 \mathrm{mag}$ of the peak of the PN luminosity function. To use this population of PNe to infer the chemical evolution of their host galaxies, it is necessary to understand the biases intrinsic to this population of PNe.

Second, planetary nebulae are the descendants of evolved stars. It is necessary to know how the nucleosynthesis associated with the evolution of the progenitor star has modified its original chemical composition, for only elements whose abundance survives this evolution unchanged are useful for studying the chemical evolution of the host galaxies. It is well-established that the stellar progenitors of $\mathrm{PNe}$ modify their original abundances of $\mathrm{He}, \mathrm{C}, \mathrm{N}$, and s-process elements, e.g., $[25,8]$. Fig. 1 implies that the processes of nucleosynthesis and dredge-up in PN progenitors appear to be approximately equally efficient in all galaxies studied so far, since the N/O ratios in their bright $\mathrm{PNe}$ span the same range of values. There is still considerable debate regarding whether $\mathrm{O}$ and $\mathrm{Ne}$ are modified. Models indicate that PN progenitors may modify their initial abundance of $\mathrm{O}$ and $\mathrm{Ne}$ slightly, though the magnitude and even the sign of the effect is unclear $[25,8]$. Observationally, the abundances of $\mathrm{O}$ and $\mathrm{Ne}$ in bright $\mathrm{PNe}$ correlate in the same 

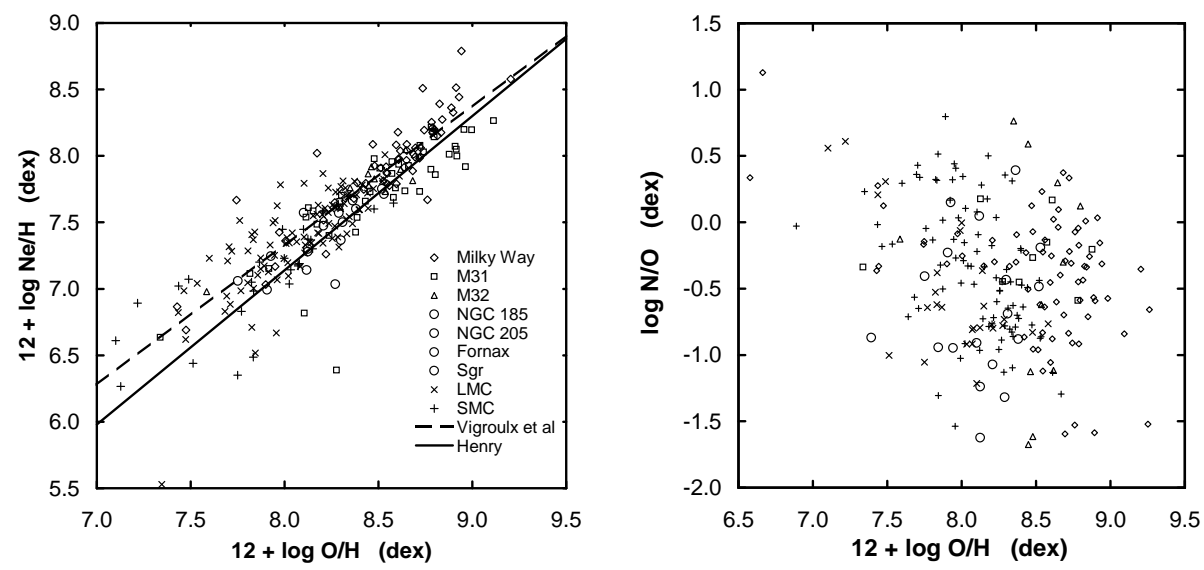

Fig. 1. Left: The oxygen and neon abundances observed in bright PNe follow the same relation as found for the ISM in star-forming galaxies $[45,13]$. The simplest interpretation is that the progenitors of bright PNe do not significantly modify either of these abundances. Right: The N/O ratios for PNe in all galaxies scatter over the same range of values, indicating that nucleosynthesis and mixing processes in the progenitor stars of bright PNe have approximately the same effect in all galaxies. The symbols have the same meaning in both figures. The data are adopted from $[40,17,36]$.

way as in giant extragalactic H II regions (Fig. 1) [45,13,40]. Type II supernovae are believed to be primarily responsible for setting this abundance ratio in the ISM, so observing the same correlation in bright $\mathrm{PNe}$ and $\mathrm{H}$ II regions implies that the progenitors of bright PNe probably do not significantly modify either their $\mathrm{O}$ or $\mathrm{Ne}$ abundances. Both $\mathrm{O}$ and $\mathrm{Ne}$ abundances in bright $\mathrm{PNe}$ should be useful probes for studying the chemical evolution of their host galaxies.

Finally, it is necessary to understand what the mean oxygen abundances in bright PNe represent. In the Magellanic Clouds, bright PNe, H II regions, and young stars all have similar oxygen abundances $[30,14,43]$. The same situation is found in NGC 6822 [44]. The agreement between stellar and nebular abundances implies that there are no serious problems with the nebular abundance scale based upon forbidden lines, since the stellar abundances are based upon permitted lines. Bright PNe and H II regions in NGC 3109, Sextans A, and Sextans B likewise have similar oxygen abundances [24,19]. Therefore, in starforming systems, in general, bright PNe have oxygen abundances very similar to those in the ISM. Fewer systems without star formation are available for similar study. The best case study is the bulge of the Milky Way. There, bright PNe have a mean oxygen abundance similar to the mean oxygen abundance in the stars $[27,40]$. This result may hold more generally for the entire PN population [6]. In the Fornax and Sagittarius dwarf spheroidals, the oxygen abundances of their PNe are typical of the younger, more metal-rich stellar populations. In Sagittarius, this population appears to dominate the mass [5]. In Fornax, the stellar population of intermediate metallicity dominates the mass [37], but For- 
nax's total stellar mass is so low that it is fortunate to observe a single bright $\mathrm{PN}$. Therefore, in systems without star formation, the oxygen abundance in the bright PNe appears to be representative of the mean oxygen abundance in the stars.

It is worthwhile trying to understand why this abundance dichotomy arises in systems with and without star formation. Many processes in stellar and galactic evolution can affect the population of bright PNe. The means by which mass is lost by a PN precursor on and immediately after the asymptotic giant branch (AGB) should affect the eventual PN morphology. In the Milky Way, the morphology of PNe correlates with their scale height above the galactic plane [9], so morphology should correlate with progenitor mass. Morphology could significantly influence PN luminosity, since it will affect the angular distribution of the nebular optical depth. There is a sensitive interplay between the time scale to evolve from the AGB to the PN phase and the nebular expansion velocity, since both affect the density of the nebular shell and its consequent optical depth and luminosity [40]. A bright PN will only be observed if the central star is hot and bright while the nebular shell is sufficiently dense. The time scale of the phase during which the central star is bright is a strong function of the mass of the central star, e.g., [3], which, in turn, is a function of the progenitor mass. The time scale during which the central star is bright also depends upon whether the it leaves the AGB burning $\mathrm{H}$ or He, e.g., [42]. Finally, the time required for a star to evolve from the AGB to the PN phase further depends upon the envelope mass remaining when the star evolves off the AGB [39], since this mass must be disposed of through either a stellar wind or nuclear burning. All of these effects may well depend upon the progenitor mass and metallicity. A key unknown is whether the evolution of the nebular shell is correlated with that of the central star $[46,33]$.

In a galactic context, chemical evolution usually ensures a correlation between the masses and metallicities of the PN progenitors. This arises through the history of star formation, which defines the mass range spanned by the PN progenitors and their number distribution [33]. In star-forming galaxies, the PN progenitors span the entire range of masses allowed. Since the death rate in a stellar population is a strong function of the population's age [29], the youngest populations can easily dominate the production of $\mathrm{PNe}$, if the star formation rate has been roughly constant (often a reasonable approximation). A high death rate alone is no guarantee that the youngest stellar populations dominate the production of bright $\mathrm{PNe}$, as the highest-mass central stars evolve so fast that it is statistically unlikely to observe such PNe [16,28]. In the LMC, the youngest stellar populations apparently produce PNe fainter than do somewhat older stellar populations [38]. In DHSs, where star formation ceased several $10^{9}$ years ago, the PN progenitors will span a small range of masses, so the death rates among the different stellar populations will be similar and each population should contribute PNe in proportion to its mass, yielding mean chemical abundances in the PN population very similar to those in the stars. These arguments are in qualitative agreement with attempts to model this process [33] and illustrate why the 


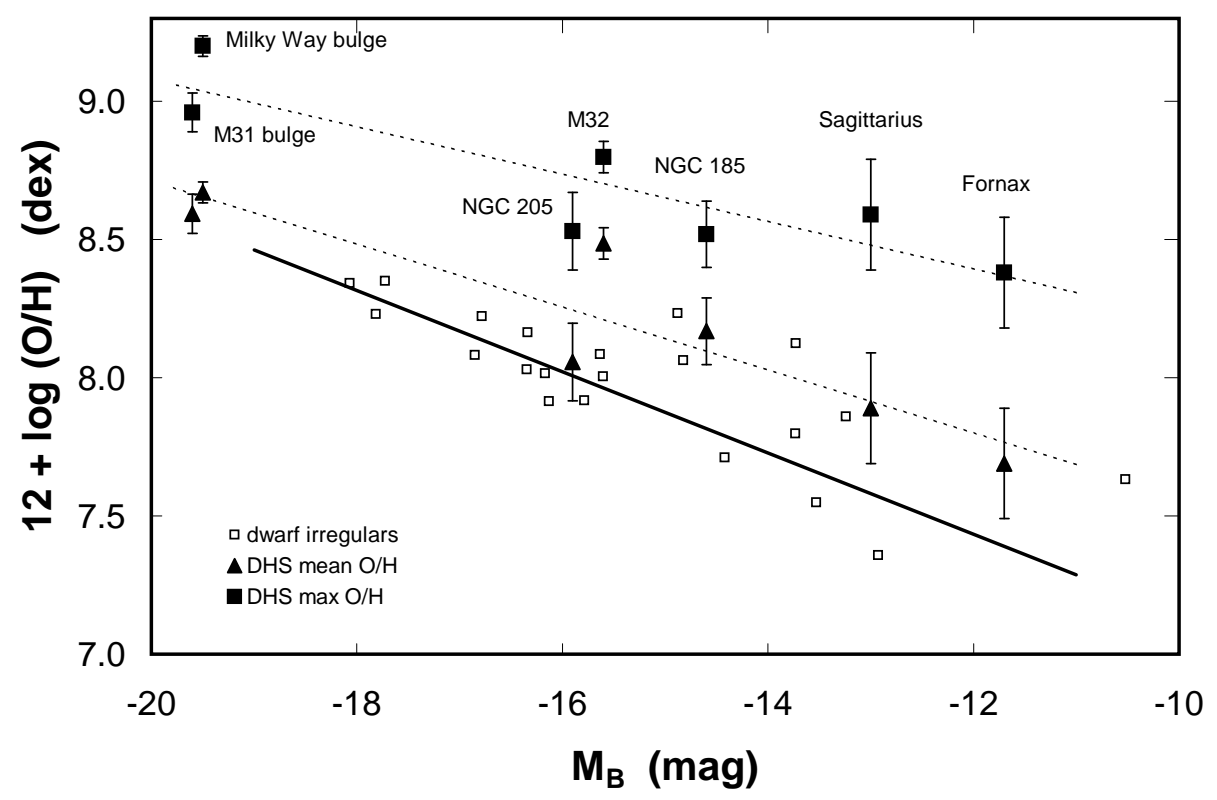

Fig. 2. This figure presents the relation between oxygen abundance and luminosity in the $B$-band for a sample of DHSs. The two dotted lines show the mean and maximum oxygen abundances found for PNe in DHSs. For reference, the same relation is shown for dwarf irregular galaxies, which is based upon oxygen abundances measured in $\mathrm{H}$ II regions, and thus should be compared with the maximum abundances observed in DHSs [31].

relations between abundances in bright $\mathrm{PNe}$ and other tracers in galaxies with and without star formation might arise.

\section{The Chemical Evolution of DHSs}

A fundamental assumption in the analysis that follows is that the bright PNe in all DHSs have mean oxygen abundances that are representative of the mean oxygen abundance in their stars, as occurs in the bulge of the Milky Way. The dwarf spheroidals Fornax and Sagittarius are the only exceptions, where we assume that their PN populations represent only their more metal-rich stellar populations and the mean oxygen abundance for their entire stellar content is reduced appropriately. The results depend upon the validity of this assumption.

DHSs follow a well-defined relation between their oxygen abundances and luminosities, as presented in Fig. 2. The relation is plotted using both the mean oxygen abundance observed in the PNe in each DHS as well as using the maximum abundance observed. The latter relation should approximate the maximum oxygen abundances found in the ISM before star formation ceased in these DHSs. 
These maximum abundances should be approximately analogous to the oxygen abundances observed in $\mathrm{H}$ II regions in star-forming galaxies [31]. In star-forming galaxies, this relation is thought to arise because galaxies of different mass have converted different fractions of their gas into stars, and so the remaining ISM has been enriched more in those galaxies with the least gas remaining [23]. This same process is believed to have occurred in DHSs, but that, at some point, the DHSs lost their gas.

DHSs must have faded since they ceased forming stars. As a result, their abundance-luminosity relation has shifted to lower luminosities over time. It is easy to illustrate that fading a dwarf irregular is insufficient of itself to reproduce the abundance-luminosity relation followed by DHSs. If a typical dwarf irregular has a colour $(B-V)=0.5 \mathrm{mag}$, then after $10^{10}$ years this galaxy will have faded by less than $2.5 \mathrm{mag}$ [11] if we approximate it as a single stellar population with a metallicity $Z=0.008$, i.e., similar to the metallicity of the LMC. The approximation of a single stellar population will exaggerate the amount of fading, since much of the light is contributed by stellar populations with redder colours that will fade less. However, 5 mag separate the abundance-luminosity relation for dwarf irregulars and the maximum abundance relation for DHSs. Therefore, fading alone cannot explain the shift of the abundance-luminosity relations for DHSs.

The abundance-luminosity relation for DHSs may also be offset if their ISM was enriched more efficiently in newly synthesized elements. Preliminary attempts to model the mean oxygen abundances and their dispersions find that it is difficult to explain simultaneously the mean abundances and their dispersions in DHSs unless DHSs were losing gas even while still forming stars [26]. As a result, the enrichment of their ISM proceeded at a faster rate than in today's dwarf irregulars because of reduced dilution of the nucleosynthetic products ejected by supernovae. The effective yield of oxygen derived for DHSs exceeds that derived for dwarf irregulars [26,23], and even that derived recently for the inner parts of spiral galaxy disks $[10,4]$, but well within the range expected from stellar nucleosynthesis calculations [20].

The helium abundances of the PNe in DHSs offer additional evidence in favour of efficient enrichment of their ISM. In Fig. 3, the helium abundances in $\mathrm{PNe}$ in different galaxies are plotted as a function of their oxygen abundances. At a given oxygen abundance, the PNe in M32 and the dwarf spheroidals, Fornax, Sagittarius, NGC 185 and NGC 205 have higher helium abundances than the PNe in the Magellanic Clouds. This effect is not so obvious for the bulges of the Milky Way or M31, but these are also the DHSs that fall closest to the abundance-luminosity relation for dwarf irregulars in Fig. 2. If the similar scatter in N/O is taken as evidence that mixing and nucleosynthesis in the PN progenitors was similar in all galaxies (Fig. 1), the cause of the high helium abundances in the DHSs should then be the result of the evolution of their host galaxies, and not the PN progenitors. Efficient enrichment of the ISM is such a process. In fact, gas outflow should affect helium enrichment more than oxygen enrichment because the production of helium is dominated by stars of lower mass (and which 


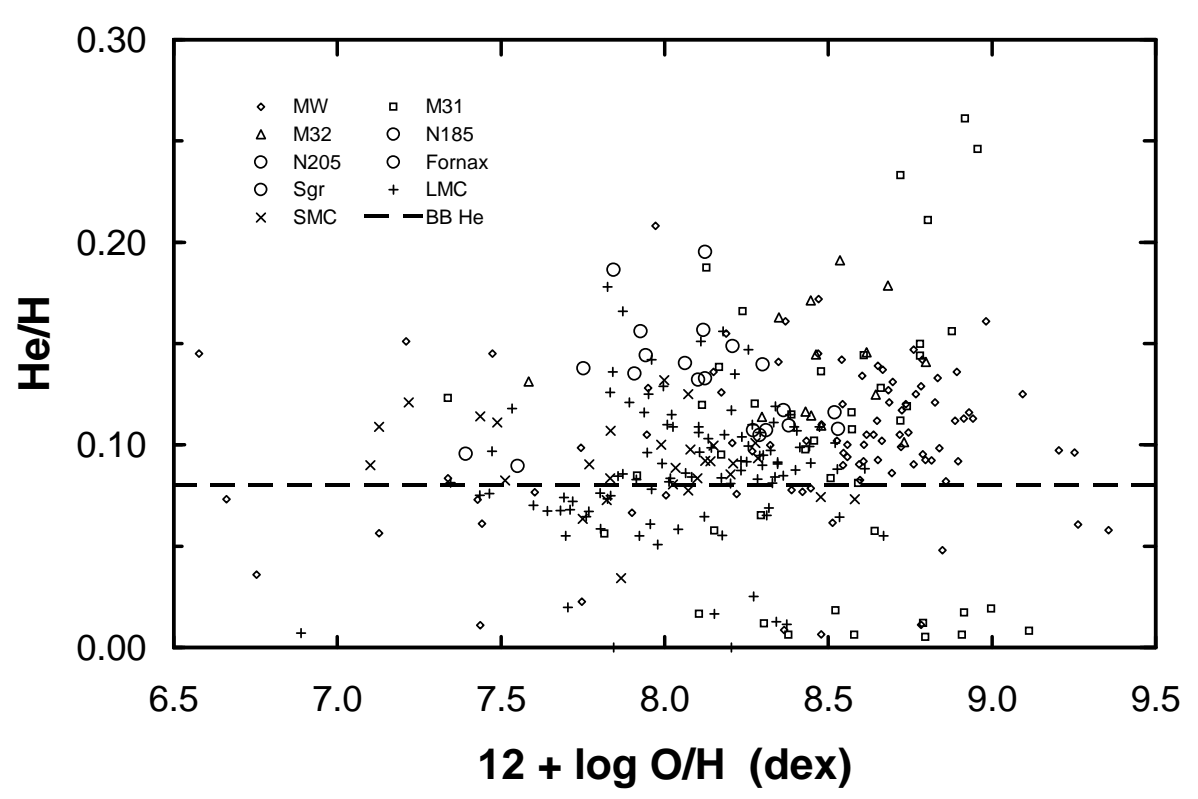

Fig. 3. Helium abundances are plotted as a function of the oxygen abundance for the $\mathrm{PNe}$ in various galaxies. At a given oxygen abundance, the helium abundance is higher in the PNe in M32 and the dwarf spheroidals than it is in the PNe in the Magellanic Clouds. Given that mixing and nucleosynthesis have the same effects upon the PN progenitors in all galaxies (Fig. 1), processes related to the evolution of the host galaxies, such as the enrichment of the ISM, should be responsible for the higher helium abundances in the DHSs. The very helium abundances (near zero) are the result of missing ionization stages. The data are adopted from $[40,17,36]$.

live longer) than is the case for oxygen [22], and so the ISM has more time to evolve (lose mass) during the production of helium.

Supernova-driven winds are commonly believed to have removed the gas from DHSs [21]. The basis for this belief is the correlation between metallicity indices and the velocity dispersion, e.g., the $\mathrm{Mg}_{2}-\sigma$ relation [2]. SNe are responsible for injecting newly-synthesized elements into the ISM, but they likewise inject large quantities of energy into the ISM. If the energy injection rate exceeds the dissipation rate, the thermal energy of the ISM increases and it may eventually exceed its gravitational binding energy. At that point, the ISM begins to flow out of the galaxy in a wind, presumably halting star formation at the same time. Massive galaxies are enriched more than low-mass galaxies since more $\mathrm{SNe}$ are required to initiate a wind. In this scenario, the chemical enrichment of a galaxy is related to the velocity dispersion of its stars only because both are controlled by the gravitational potential. Since oxygen is produced by core collapse $\mathrm{SNe}$, the oxygen abundance is a faithful tracer of the energy they injected 


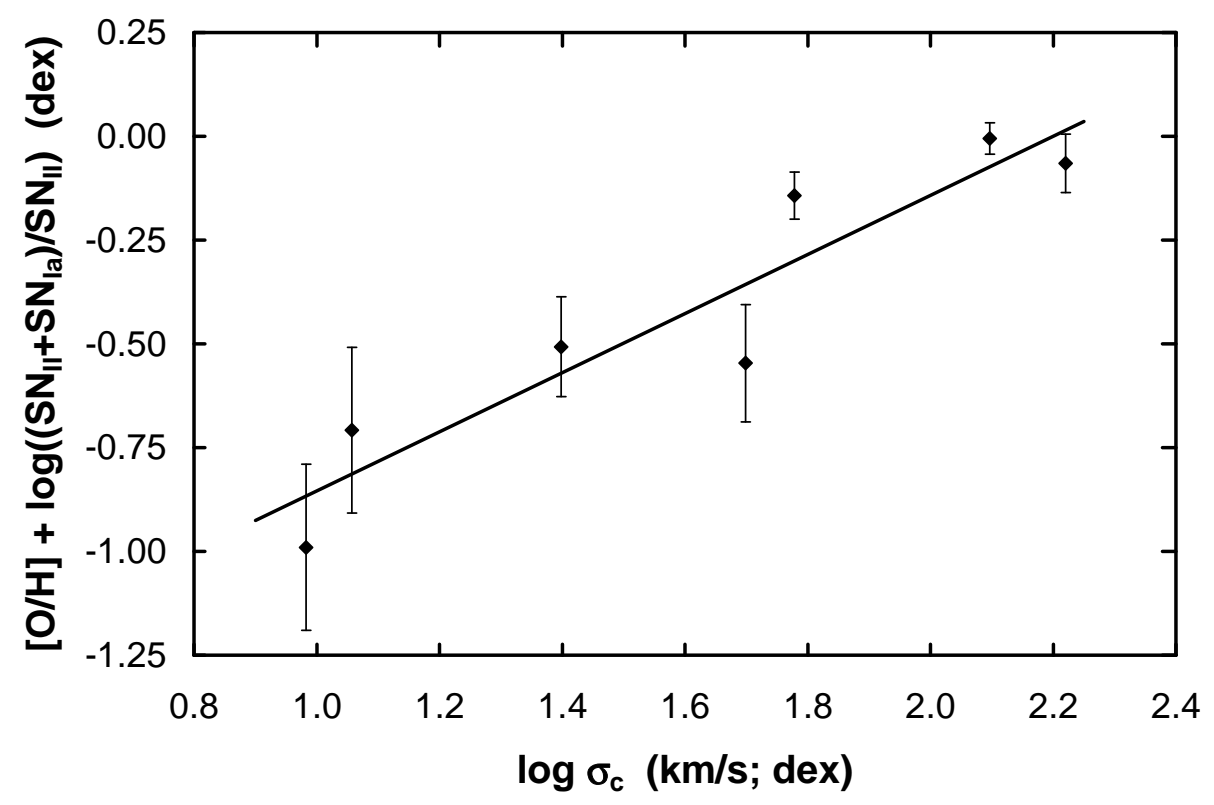

Fig. 4. Oxygen abundances are plotted versus velocity dispersion in DHSs. The oxygen abundances have been corrected to account for the energy injection from type Ia SNe. This relation arises naturally if supernova-driven winds are the mechanism that terminates chemical evolution in DHSs.

into the ISM. However, star formation in DHSs may have extended over a period long enough that type Ia supernovae also contributed energy and matter to the ISM. One may correct the oxygen abundances to account for the contribution of type Ia SNe using the $\mathrm{O} / \mathrm{Fe}$ ratio observed in stars [34], since type Ia $\mathrm{SNe}$ contribute significantly to iron enrichment, but not to oxygen enrichment. Fig. 4 presents these corrected oxygen abundances plotted as a function of the velocity dispersion in their host galaxy. A correlation is found that is consistent with expectations if the chemical evolution of DHSs was terminated by the action of supernova-driven winds.

The role of mergers in the formation of DHSs is difficult to discern from these results. For gas-rich, major mergers (components with similar masses), the chemical evolution is changed very little compared to a monolithic formation scenario, since a large fraction of the star formation occurs after assembling the baryonic mass. Major mergers that are gas-poor would flatten the abundanceluminosity relations in Fig. 2 and the abundance-velocity dispersion relation in Fig. 4, for most of the stars would form in a shallower gravitational potential than that in which they now reside. The slope and not the intercept of the relations is affected because the smallest DHSs considered here are too small to be the products of mergers themselves. Minor mergers (components with 
very different masses) would not be distinguishable in Figs. 2 and 4 regardless of their gas content. Clearly, the only scenario ruled out is the assembly of large DHSs entirely from a large number of small, gas-poor systems. This would produce a DHS that falls below the relations in Figs. 2 and 4, which has not been observed so far. Basically, one would find the same oxygen abundances in all DHSs, regardless of mass.

\section{Conclusions}

Two observations indicate that bright PNe in DHSs should be useful probes to infer the chemical evolution of these galaxies. First, the precursors of bright $\mathrm{PNe}$ do not significantly modify their original oxygen abundances. Second, the mean oxygen abundance measured in bright $\mathrm{PNe}$ appears to be representative of the average oxygen abundance for the entire stellar population in DHSs. Nonetheless, there are numerous uncertainties concerning the production of populations of bright PNe and any advance in quantifying the processes involved, at either the stellar or galactic level, will improve the utility of PNe for studying the chemical evolution of DHSs.

DHSs are found to follow a relation between oxygen abundance and luminosity. This relation is shifted to higher abundances than that followed by dwarf irregular galaxies, independently of whether the mean or maximum oxygen abundances for the DHSs are considered. Fading appears to be insufficient to explain the shift. DHSs appear to have incorporated their nucleosynthetic production into subsequent stellar generations more efficiently than did dwarf irregulars.

\section{References}

1. Bender, R., Burstein, D., \& Faber, S. M. 1992, ApJ, 399, 462

2. Bender, R., Burstein, D., \& Faber, S. M. 1993, ApJ, 411, 153

3. Blcker, T. 1995, A\&A, 299, 755

4. Bresolin, F., Garnett, D. R., \& Kennicutt, R. C., Jr. 2004, ApJ, in press; also astro-ph/0407065

5. Cole, A. 2001, ApJ, 559, L17

6. Exter, K. M., Barlow, M. J., \& Walton, N. A. 2004, MNRAS, 349, 1291

7. Faber, S. M., ApJ, 179, 423

8. Forestini, M., \& Charbonnel, C. 1997, A\&AS, 123, 241

9. Garcia-Segura, G., Franco, J., López, J. A., Langer, N., \& Różyczka, M. 2002, RMAAC, 12, 117

10. Garnett, D. R., Kennicutt, R. C., Jr., \& Bresolin, F. 2004, ApJ, 607, L21

11. Girardi, L., Bressan, A., Bertelli, G., \& Chiosi, C. 2000, A\&AS, 141, 371

12. Greenawalt, B., Walterboss, R. A. M., \& Braun, R. 1997, ApJ, 483, 666

13. Henry, R. B. C. 1989, MNRAS, 241, 453

14. Hill, V., Andrievsky, S., \& Spite, M. 1995, A\&A, 293, 347

15. Izotov, Y. I., \& Thuan, T. X. 1998, ApJ, 500, 188

16. Jacoby, G. H. 1989, ApJ, 339, 39

17. Jacoby, G. H., \& Ciardullo, R. 1999, ApJ, 515, 169 
18. Jenner, D. C., Ford, H. C., \& Jacoby, G. H. 1979, ApJ, 227, 391

19. Kniazev, A. Y., Grebel, E. K., Pramskij, A. G., \& Pustilnik, S. A. 2004, these proceedings

20. Köppen, J., \& Arimoto, N. 1991, A\&AS, 87, 109

21. Larson, R. B. 1974, MNRAS, 169, 229

22. Maeder, A. 1992, A\&A, 264, 105

23. Lee, H., McCall, M. L., Kingsburgh, R. L., Ross, R., \& Stevenson, C. C. 2003, AJ, 125,146

24. Magrini, L., Perinotto, M., Corradi, R., Leisy, P., \& Mampaso, A. 2004, these proceedings

25. Marigo, P., Bressan, A., \& Chiosi, C. 1996, A\&A, 313, 545

26. McCall, M. L., \& Richer, M. G. 2003, in IAU Symp. 209, Planetary Nebulae: Their Evolution and Role in the Universe, eds. S. Kwok, M. Dopita, and R. Sutherland (ASP: San Francisco), 583

27. McWilliam, A., \& Rich, R. M. 1994, ApJS, 91, 749

28. Méndez, R. H., \& Soffner, T. 1997, A\&A, 321, 898

29. Renzini, A., \& Buzzoni, A. 1986, in Spectral Evolution of Galaxies, eds. C. Chiosi and A. Renzini (D. Reidel Publishing Co.: Dordrecht), 195

30. Richer, M. G. 1993, ApJ, 415, 240

31. Richer, M. G., \& McCall, M. L. 1995, ApJ, 445, 642

32. Richer, M. G., \& McCall, M. L. 2004, in preparation

33. Richer, M. G., McCall, M. L., \& Arimoto, N. 1997, A\&AS, 122, 215

34. Richer, M. G., McCall, M. L., \& Stasińska, G. 1998, A\&A, 340, 67

35. Richer, M. G., Stasińska, G., \& McCall, M. L. 1999, A\&AS, 135, 203

36. Roth, M. M., Becker, T., Kelz, A., \& Schmoll, J. 2004, ApJ, 603, 531

37. Saviane, I., Held, E. V., \& Bertelli, G. 2000, A\&A, 355, 56

38. Stanghellini, L. 2004, these proceedings

39. Stanghellini, L., \& Renzini, A. 2000, ApJ, 542, 308

40. Stasińska, G., Richer, M. G., \& McCall, M. L. 1998, A\&A, 336, 667

41. Tolstoy, E., Venn, K. A., Shetrone, M., Primas, F., Hill, V., Kaufer, A., \& Szeifert, T. 2003, AJ, 125, 707

42. Vassiliadis, E., \& Wood, P. R. 1994, ApJS, 92, 125

43. Venn, K. A. 1999, ApJ, 518, 405

44. Venn, K. A., Lennon, D. J., Kaufer, A., McCarthy, J. K., Przybilla, N., Kudritzki, R. P., Lemke, M., Skillman, E. D., \& Smart, S. J. 2001, ApJ, 547, 765

45. Vigroulx, L., Stasińska, G., \& Comte, G. 1987, A\&A, 172, 15

46. Villaver, E., Manchado, A., \& García-Segura, G. 2002, ApJ, 581, 1204

47. Walsh, J. R., Walton, N. A., Jacoby, G. H., \& Peletier, R. F. 1999, A\&A, 346, 753 